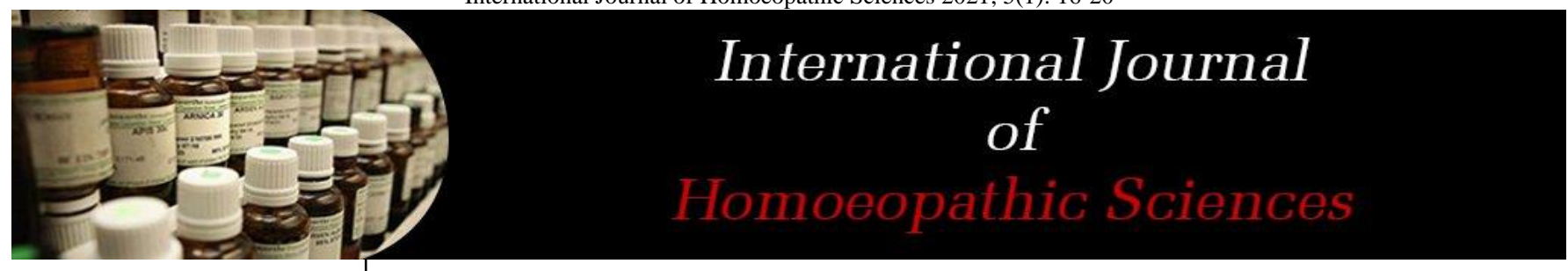

E-ISSN: 2616-4493 P-ISSN: 2616-4485 www.homoeopathicjournal.com IJHS 2021; 5(1): 16-20 Received: 12-10-2020 Accepted: 10-12-2020

Greeshma IK

Postgraduate Scholar, Department of Practice of Medicine, National Homoeopathy Research Institute in Mental Health, Kottayam, Kerala, India

\section{Dastagiri P}

Research Officer/Scientist-I, National Homoeopathy Research Institute in Mental Health, Under Central Council for Research in Homoeopathy, Ministry of AYUSH,

Kottayam, Kerala, India
Corresponding Author: Greeshma IK

Postgraduate Scholar, Department of Practice of Medicine, National Homoeopathy Research Institute in Mental Health, Kottayam, Kerala, India

\section{A review of the effectiveness of homoeopathy in the management of chronic leg ulcers}

\section{Greeshma IK and Dastagiri P}

DOI: https://doi.org/10.33545/26164485.2021.v5.i1a.285

\begin{abstract}
Background and Objectives: Any ongoing injury which shows no inclination to heal following 3 months of suitable therapy or in a time of a year the injury is not completely healed comprise chronic leg ulcers. Venous, Arterial and Neuropathic ulcers are the major types of chronic leg ulcers and factors like smoking, obesity, Diabetes mellitus \& increased age boost up the incidence of ulceration. Expanded rate of leg ulcers, monetary weight, and recurrence of the ulcer which increases the degree of Homeopathy. The present review comprises the literature on the Homoeopathic approach in managing the various chronic leg ulcers.

Materials and Methods: A literature search was conducted in databases like PubMed, Google Scholar, Wiley online library, Springer, Scopus, AYUSH portal, to collect all relevant research studies, review article, case series \& case reports, published from 1990 until July 2020. This research was categorised by study type and assessed according to study design with their clinical outcomes.

Results: Out of these 5 studies, 3 are case studies \& one is a prospective observational study and one is a randomized clinical trial and it shows that chronic leg ulcers have marked improvement by the use of Homoeopathic medicine both internally and externally.

Conclusion: Homoeopathy is effective in the management of chronic leg ulcers mainly diabetic foot ulcers and varicose ulcers. A combination of both internal and external medicines also showed excellent results in treating this illness. There is only limited evidence available to show the effectiveness of Homoeopathy and more studies needed for the same.
\end{abstract}

Keywords: Chronic leg ulcer, varicose ulcer, diabetic foot ulcer, homoeopathy

\section{Introduction}

Damage to the skin which occurs below the level of knee persisting more than 6 weeks \& showing no tendency to heal after 3 or more months is known as chronic leg ulcer (CLU). Smoking, obesity, Diabetes mellitus, increased age, etc. enhancing the risk for atherosclerotic occlusion which in turn increases the incidence of ulceration ${ }^{[1]}$. This type of painful debilitating leg ulcers may greatly reduce the quality of life of the patient. Accurate diagnosis and treatment of underlying causes are important for the successful treatment of chronic leg ulcer ${ }^{[2]}$. Leg ulcers are very often associated with pain, itching, very offensive discharges, swelling of the leg, and decrease in the patient's mobility leading to both psychological and physical aspects like restriction in occupational and domestic activities, feeling social isolation, having a pessimistic view about future, anger, depressed feeling, decreased will power, hopelessness, sense of uncleanliness and low self-esteem due to altered their body image ${ }^{[3,4,5]}$. The prevalence of CLU across the world ranges from $1.9 \%$ to $13.1 \%$. In India, very few studies have been conducted on the epidemiology of ulcers one of the studies estimated that 4.5 per 1000 population has been suffering from this disorder. The incidence of acute wounds was more than double at 10.5 per 1000 population ${ }^{[6,7]}$. It may very often affect $0.6-3 \%$ over 60 years aged and increasing to over 5\% aged over 80 years [8]. According to the Wound Healing Society, in the US about $15 \%$ of older adults suffer from chronic wounds, including predominantly venous stasis ulcers, pressure ulcers (bedsores), and diabetic (neuropathic) foot ulcers. Every year 2 to 3 million Americans are diagnosed with various types of chronic wounds ${ }^{[9]}$. The estimates of the annual incidence of leg ulcers in the UK and Switzerland are 3.5 and 0.2 per 1000 individuals, respectively. The prevalence of vascular ulcers in the US is estimated at 500,000 to 600,000 and increases with age ${ }^{[10,11]}$. Based on aetiology, CLUs are mainly 3 types: venous ulcers, arterial ulcers, and neuropathic ulcers. Among the ulcers, $81 \%$ are due to venous disease and it represents the most advanced stage of chronic venous insufficiency, $16.3 \%$ are arterial ulcers ${ }^{[3]}$ and and 


\section{$15 \%$ of diabetic foot ulcer}

[1].

A study from India shows that $40 \%$ leprosy, $23 \%$ diabetes, $11 \%$ venous disease, and $13 \%$ trauma are the reasons for ulcers in lower extremity ${ }^{2}$. Moreover in India, most of the chronic leg ulcers are due to venous aetiology and sometimes caused by Filariasis, Tuberculosis, and Leprosy ${ }^{\text {[2] }}$. Finally, the main causes of chronic leg ulcers are venous and arterial insufficiency, neuropathy, diabetes, or a combination of these factors ${ }^{[12]}$.

There are only a few studies that have been conducted in Homoeopathy on the management of chronic leg ulcers which are mostly varicose ulcers and diabetic foot ulcers. Most of the studies are case series and evidence-based clinical studies. The increased incidence of leg ulcers, economic burden, and recurrence of ulcers increase the scope of Homoeopathy in this regard. The present review comprises the literature on the Homoeopathic approach in managing the various chronic leg ulcers.

\section{Aim and objectives}

This study aimed to know the effectiveness of Homoeopathy in the management of Chronic Leg ulcers \& also aimed to develop a strategy for further scientific research in Homoeopathy.

\section{Methods}

Literature search and identification of studies

A comprehensive literature search was conducted using the keyword chronic leg ulcer, varicose ulcer, arterial ulcer, diabetic ulcer, and Homoeopathy in databases like Pub Med, Google Scholar, Wiley online library, Springer, Scopus, and AYUSH portal to collect all relevant review, research articles, case series \& case reports published until July 2020 indicating the scope of Homoeopathy in managing chronic leg ulcers. It was limited to the English language of publication (Excluding non-peer-reviewed journals). The search results were narrowed down to study the effectiveness of Homoeopathic drugs either individually or in combination with another drug in managing the chronic leg ulcer. All references were screened for eligibility based on their abstract of full text, and data were extracted by the second author. After extraction of the data, a total, five $(n=5)$ studies were found to be eligible as per the criteria.

\section{Result of review ${ }^{[13-17]}$}

Out of the search results using keywords, the first abstracts of studies were analyzed whether they tested the efficacy of Homoeopathic medicine in managing chronic leg ulcers. Only 5 studies were selected based on their abstract due to the limited number of literature. Diabetic gangrene has been excluded from this study. Out of these 5 studies, 3 are case studies and one is a prospective observational study and one is a randomized clinical trial. Full text and abstract of these 5 studies were analyzed for its study design, methodology, Homoeopathic medicine prescribed, and their outcome. (Figure 1)

\section{The approach of homoeopathy in diabetic foot ulcer}

A prospective observational study was conducted by the Central Council for Research in Homoeopathy, Hyderabad from October 2005 to September 2009. 63 cases were enrolled in the study. Using the rubrics 'Diabetes mellitus' and 'Non-healing Ulcer' in The Complete Repertory fifteen pre-defined Homoeopathic medicines were selected. These were: Sulphur, Silicea, Lycopodium, Arsenium album, Lachesis, Phosphorus, Sepia, Phosphoricum Acidum, Opium, Mercurius solubilis, Pulsatilla, Secale cornutum, Calcarea carbonicum, Plumbum metallicum, and Rhus Toxicodendron. Based on the totality of symptoms of each patient, similimum was selected from this shortlist of medicines. The outcome was assessed by a reduction in diabetic foot ulcer symptom score. Out of fifteen medicine, 9 medicines prescribed for the patients. 5 most useful medicines found in this study were Silicea, Sulphur, Lycopodium, Arsenicum album, and Phosphorus. In 95.5\% of the cases in which Silicea administered showed an improvement, Sulphur in $90.9 \%$ of cases, Lycopodium and Arsenicum album in $100 \%$, and Phosphorus in $75 \%$ cases showed improvement ${ }^{7}$. Most of the cases were cured within 3 months and few at the end of the study ${ }^{[13]}$.

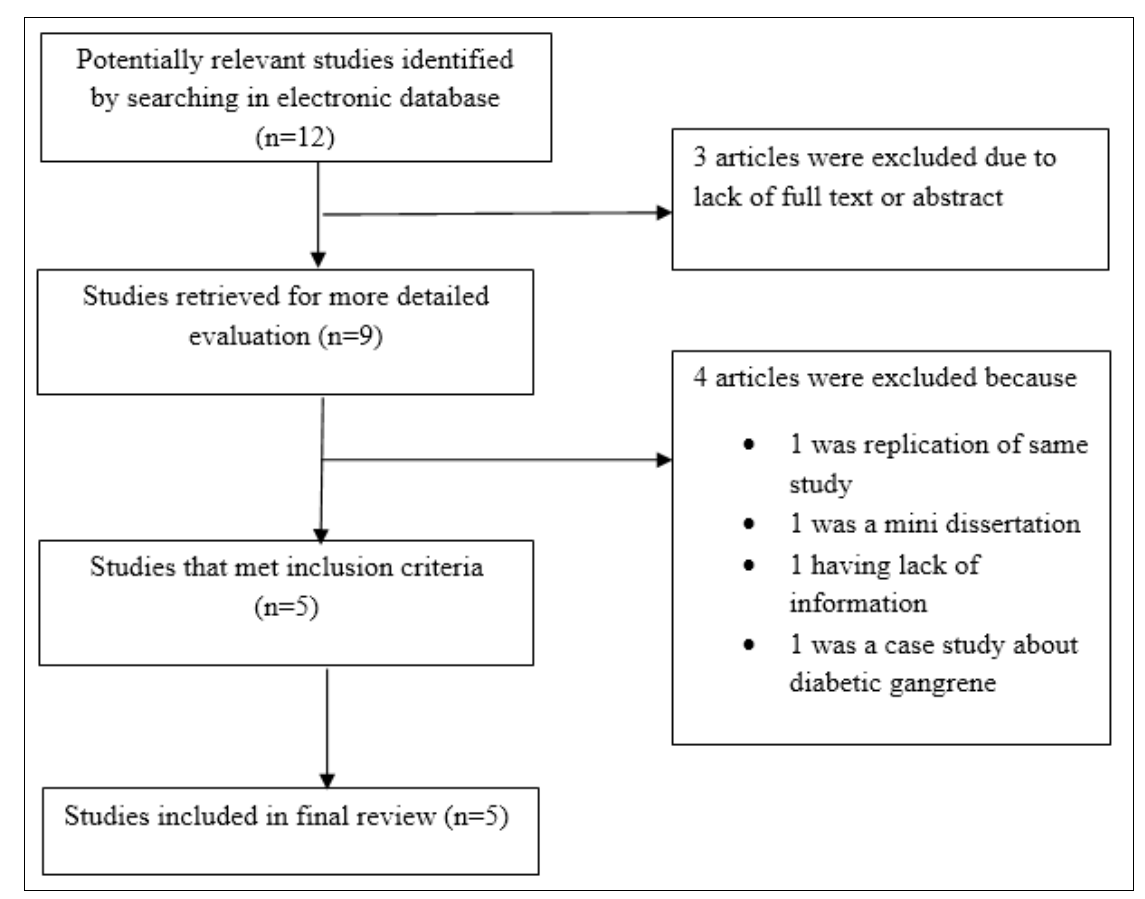


Fig 1: Diagram outlining literature search results

Persis Gadde et al. reported that a case of Diabetic foot ulcer received Homoeopathic treatment and responded well with improvement both in the healing of ulcer and general health. A 70-year-old female patient who was a 43 years known diabetic presented with an ulcer in the sole of the left foot for 4 years. The ulcer was very painful to touch and exposed to cold with offensive discharge. Based on the totality of symptoms Hepar sulph 30 three doses once in a week was prescribed on alternate months for 3 months and then Hepar sulph 200 three doses weekly once for 4 months and later Hepar sulph $1 \mathrm{M}$ one dose was given. Externally calendula mother tincture was used. After 10 months of treatment with hepar sulph, there was a marked reduction of localized infection and marked healing of the ulcer. There was an $85.2 \%$ improvement as per the DFU assessment scale and also a reduction in the blood sugar level. The score of Modified Naranjo Criteria in this study was 9 and it shows definite causal attribution of Homoeopathic treatment with the outcome ${ }^{[14]}$.

\section{The approach of homoeopathy in the varicose ulcer}

An evidence-based case study by Chandranath Das states that Homoeopathic medicine is very effective in treating long-standing and recurring varicose ulcers. An ulcer on the right leg near medial malleolus with varicosities was treated with Natrum mur 200/2 doses for 5 days and calendula mother tincture for dressing the ulcer. According to the indications, Arnica 200/2 doses once daily prescribed for 2 months then Ars Alb 30/8 doses twice in a day for 6 days and Ipecac 30/2 doses once in a day for 1 month. After that Natrum Sulph 200/2 doses daily once for 8 days, Lycopodium $1 \mathrm{M} / 1$ dose for 11 days, and Calcarea sulph $30 / 4$ doses two and half months given and finally Silicea 200/2 doses daily were prescribed to cure the case. After 5 months of treatment with Homoeopathic medicines for varicose ulcer healed completely ${ }^{[15]}$. A case study by Angela $\mathrm{V}$ et al. in 3 cases of venous leg ulcers shows that Novel Wound Healing Powder, a formulation of Calendula officinalis L (SI $0.1 \%$ vol/wt) plus Arnica montana L (SI $0.01 \% \mathrm{vol} / \mathrm{wt})$ and inactive ingredients Mentha arvensis (mint, $90 \%$ wt/wt) plus Santalum album (sandalwood, SI 10\% wt/wt) is effective for the treatment of Venous Leg Ulcers. In combination with the use of compression stockings or wraps, this powder was found to reduce wound pain and accelerate the healing of slow-toheal wounds. It absorbed excessive wound exudates, sealed the wound from bacteria, helped maintain moisture balance, and reduced the malodors ${ }^{[16]}$.

\section{The approach of homoeopathy in leg ulcer}

A randomized clinical trial conducted by B Garrett et al. finds out that Homoeopathic medicine Sulphur. Silicea and Carbo vegetabilis in $6^{\text {th }}$ centesimal potency is effective in the treatment of leg ulcers. In this study, 23 patients from clinics at Southport and Formby Hospital, Southport, and Queen Victoria Hospital were selected and randomly allocated to the test group (nine patients) or control group (seven patients). Patients who unwilling to be treated homoeopathically, or for whom the homoeopathic treatment would have been contraindicated, were allocated to a placebo group (seven patients). Conventional medicine and nursing care were continued during the study period. Ulcer measurements over the mean treatment period of 4.2 weeks show that the addition of SSC to the patients' treatments enhanced ulcer healing ${ }^{[17]}$.

\section{Discussion}

In the prospective observational study, CCRH has followed the individualistic approach, which is one of the cardinal principles of Homeopathy, with predefined drugs based on the complete repertory. The significance of improvement was assessed by validated DFU symptom score before and after the intervention, and most of the cases were cured in three months without any complications ${ }^{[13]}$.

In the Peresis et al., evidence base study, the diabetic foot Ulcer was explicitly treated by both internal and external intervention of Homoeopathic medicines as Hepar. sul and Calendula mother tincture for dressing the ulcer respectively. With this intervention, the author had observed $85.2 \%$ improvement in the DFU scale and also a reduction in blood sugar level, further this case improvement was affirmed by the Modified Naranjo criteria's causal attribution score that with the Homoeopathic intervention the results were obtained ${ }^{[14]}$.

Another evidence-based, long-standing, and recurring venous ulcer, treated successfully by Chandranath Das with Homoeopathic intervention both externally and internally as mentioned above with different medicines at different intervals based on the indication of the totality of symptoms of the patient, in 5 months. The deficit, in this case, was the author didn't apply any prognostic scales before and after intervention except photographs of the ulcer ${ }^{[15]}$

The case series of Angela et al. showed favourable results in three cases of venous ulcer with, the combination of Calendula, Arnica, Mentha, and Santalum album in reducing the pain, accelerating the healing process without any complications like exudates, malodors from the ulcers [16]. The usage of this combination of drugs has contradicted the single medicine administration the cardinal principle of Homoeopathy which has been explained from aphorism 272-.274 in $6^{\text {th }}$ edition of Organon of medicine ${ }^{[18]}$.

Garette et al., conducted a gold-standard randomized controlled trial and the final inference showed that the addition of Sulphur, Silicea, Carbo. Vegetables (SSC)-6C potency accelerates the ulcer healing in 4.2 weeks meantime, without any adverse effects ${ }^{[17]}$. Once again it was notified that this method was against the strategic principle of single medicine prescription. Assumed that maybe the medicine has an affinity towards the varicosities, the tendency to healing ulcers will direct them to select the remedies. Furthermore, the sample size is small to get explicit information, but the statistical data on a pathologic basis is truly appreciable. Homoeopathy follows strictly individualization in the selection of the remedy on basis of the totality of symptoms including the disease pathology. In the $6^{\text {th }}$ edition of Organon of Medicine, aphorism 185-203, Hahnemann emphasized that ulcers are caused by either local or internal causes. After excluding the surgical and injuries, the remaining ulcers are of Psoirc origin. If we, treated externally, probably we might not assess the exact prognosis of the external ulcer and its internal disease. He cautioned us to treat these cases judiciously with internal medication only.

\section{Conclusion}

Homoeopathy is powerful in the treatment of chronic leg 
ulcers predominantly diabetic foot ulcers and varicose ulcers. Different studies report the effect of Homeopathic medication in the treatment of leg ulcers and related indications. A blend of Homeopathic medications and external applications additionally shows great outcomes in treating persistent leg ulcers. Just a set number of confirmations is accessible to show the adequacy of Homeopathy and more investigations are required in a similar field. To strengthen the effectiveness of Homoeopathy in Chronic Leg Ulcers, we need large sample size studies with standard protocols based on diagnostic and prognostic parameters.

Table 1: Summary of studies

\begin{tabular}{|c|c|c|c|}
\hline & Evidence Grade & Evidence B & Evidence C \\
\hline Outcome & $\begin{array}{c}\text { 95.5\% of the cases in which Silicea regulated } \\
\text { demonstrated improvement, Sulphur in 90.9\% } \\
\text { of cases, Lycopodium and Arsenicum collection } \\
\text { in 100\%, and Phosphorus in 75\% cases } \\
\text { indicated improvement Based on n totality of } \\
\text { symptoms }\end{array}$ & $\begin{array}{c}\text { Based on the totality of symptoms Hepar sulph 30, 200 \& } \\
\text { 1M given serially\& calendula mother tincture externally. 10 } \\
\text { months of treatment shows the stamped decrease of localized } \\
\text { infection, healing of the ulcer \& 85.2\% improvement in DFU } \\
\text { evaluation scale \& decrease in the glucose level. The score } \\
\text { of Modified Naranjo Criteria was 9, shows causal attribution } \\
\text { of Homeopathic treatment with the result }\end{array}$ \\
\cline { 2 - 5 } Summary \\
of studies
\end{tabular}

Table 2: Summary of studies

\begin{tabular}{|c|c|c|c|c|}
\hline Evidence Grade & Evidence Grade C & Evidence Grade C & Evidence Grade B \\
\hline Outcome & $\begin{array}{c}\text { According to indications Natrum } \\
\text { mur 200, Arnica 200, Ars Alb 30 \&, } \\
\text { Ipecac 30, Natrum Sulph 200, } \\
\text { Lycopodium 1M and Calcarea sulph } \\
\text { gave and finally Silicea 200 was } \\
\text { prescribed to cure the case }\end{array}$ & $\begin{array}{c}\text { The combined use of compression stockings or wraps } \\
\text { and Novel Wound Healing Powder was found to } \\
\text { reduce wound pain and accelerate the healing of } \\
\text { slow-to-heal wounds. Also, it absorbed excessive } \\
\text { wound exudates, sealed the wound from bacteria, } \\
\text { helped maintain moisture balance and reduced the } \\
\text { malodor }\end{array}$ & $\begin{array}{c}\text { addition of SSC to the } \\
\text { patients' treatments } \\
\text { enhanced ulcer healing }\end{array}$ \\
\cline { 2 - 6 } Summary \\
of studies
\end{tabular}

\section{Reference}

1. Agale SV. Chronic leg ulcers: epidemiology, aetiopathogenesis, and management. Ulcers $2013 \mathrm{Apr}$ 22; 2013. Last accessed on 08.03.2020.

2. Dogra S, Sarangal R. Summary of recommendations for leg ulcers. Indian dermatology online journal 2014;5(3):400. Last accessed on 07.03.2020.

3. Herber OR, Schnepp W, Rieger MA. A systematic review on the impact of leg ulceration on patients' quality of life. Health and quality of life outcomes
2007;5(1):44. Last accessed on 03.11.2020.

4. Miraj AKA, Rahaman HNA, Rahman MM, Khan MSU. Impact of Leg Ulcers on Quality of Life: Financial, Social, and Psychological Implications among the Patients Attending OPD of Vascular Surgery: A Study in Bangabandhu Sheikh Mujib Medical University, Dhaka, Bangladesh. British Journal of Research 2020;7(1:51):11-17. Last accessed on 03.11.2020.

5. Phillips T, Stanton B, Provan A, Lew R. A study of the 
impact of leg ulcers on quality of life: financial, social, and psychologic implications. Journal of the American Academy of Dermatology 1994;31(1):49-53. Last accessed on 03.11.2020.

6. Narwade P, Saxena D, Wasnik N, Akhtar M. Non diabetic chronic leg ulcers: etiology and management. Int Surg J 2019;6:2070-3. Last accessed on 03.11.2020.

7. Shukla VK, Ansari MA, Gupta SK. Wound healing research: a perspective from India. International Journal of Lower Extremity Wounds 2005;1;4(1):7-9. Last accessed on 03.11.2020.

8. Rayner R, Carville K, Keaton J, Prentice J, Santamaria N. Leg ulcers: atypical presentations and associated comorbidities. Wound Practice and Research 2009;17(4):168-85. Last accessed on 03.11.2020.

9. Cheng CF, Sahu D, Tsen F, Zhao Z, Fan J, Kim R et al. A fragment of secreted Hsp90 $\alpha$ carries properties that enable it to accelerate effectively both acute and diabetic wound healing in mice. The Journal of clinical investigation 2012;1;122(2):779. Last accessed on 03.11.2020.

10. Rahman GA, Adigun JA, Fadeyi A. Epidemiology, etiology and treatment of chronic leg ulcer: experience with sixty patients. Annals of African Medicine 2010;9(1). Last accessed on 03.11.2020.

11. Mekkes J, Loots MA, Van Der Wal AC, Bos JD. Causes, investigation and treatment of leg ulceration. British Journal of dermatology 2003;148(3):388-401. Last accessed on 03.11.2020.

12. Langer V. Leg ulcers: an Indian perspective. Adi BS. Efficacy of homoeopathic medicines in chronic low back pain: A clinical study. International Journal of Alternative and Complementary Medicine 2020,17-20. Indian dermatology online journal 2014;5(4):535. Last accessed on 07. 03.2020.

13. Nayak C, Singh V, Singh K, Singh H, Gupta J, Ali MS et al. A prospective observational study to ascertain the role of homeopathic therapy in the management of diabetic foot ulcers. Indian Journal of Research in Homoeopathy 2012;1;6(2):22. Last accessed on 07. 10.2020

14. Gadde P, Narasimhulu D, Rompicherla KG. Integrative management of diabetic foot ulcers with Homoeopathy and standard care. Indian Journal of Research in Homoeopathy 2018;1;12(3):180. Last accessed on 22.07.2020

15. Das C. Evidence-based Homoeopathy in treating longstanding and recurring venous ulcer-A prospective and interventional study, International Journal of Pharmaceutical Sciences and Research 2013;1;4(8):3266-3269. Last accessed on 09. 03.2020

16. Ghatnekar AV, Elstrom T, Ghatnekar GS, Kelechi T. Novel wound healing powder formulation for the treatment of venous leg ulcers. The Journal of the American College of Certified Wound Specialists 2011;1;3(2):33-41. Last accessed on 09. 03.2020

17. Garrett B, Harrison PV, Stewart T, Porter I. A trial of homoeopathic treatment of leg ulcers. Journal of Dermatological Treatment 2009;12:115-117. Last accessed on 09. 03.2020.

18. Hahnemann S. Organon of medicine student's Economy edition, 6th edition, translated by William Boericke MD, Reprint Edition, B Jain Publishers, New Delhi 1996, 296-297. 